\title{
Lumbar Erector Spinae Plane Block for Posterior-Superior Iliac Spine Bone Graft Site Pain in Patients Undergoing Occipitocervical and C1-C2 Fusion for Atlantoaxial Dislocation/Odontoid Fracture-A Case Series
}

\author{
Ramamani Mariappan ${ }^{1}$ \\ Karen Ruby Lionel ${ }^{1}$

\section{Edmond Jonathan Gandham²} \\ ${ }^{1}$ Department of Neuroanaesthesia, Christian Medical College, \\ Vellore, Tamil Nadu, India \\ ${ }^{2}$ Department of Neurological Sciences, Christian Medical College, \\ Vellore, Tamil Nadu, India \\ ${ }^{3}$ Department of Anaesthesia, Christian Medical College, Vellore, \\ Tamil Nadu, India
}

J Neurosci Rural Pract:2021;12:213-216
Sam Jenkins Stephenson ${ }^{3}$

Noble E. Cherian ${ }^{3}$

\author{
Address for correspondence Karen Ruby Lionel, MD, DM, \\ Department of Neuroanaesthesia, Christian Medical Collage, \\ Vellore 632004, Tamil Nadu, India (e-mail: kanjacob@gmail.com).
}

\begin{abstract}
Pain at the autologous bone graft site from the posterior-superior iliac spine (PSIS) is severe enough to affect the postoperative ambulation. It adds to the morbidity of the surgical procedure. Inadequate pain management at the graft site not only affects the postoperative recovery but also can lead to chronic pain. We report the use of ultrasound (US)-guided lumbar erector spinae plane block (ESPB), to deliver effective analgesia for this pain. Patients who underwent occipitocervical fusion (OCF) and C1C2 fusion using PSIS for atlantoaxial dislocation (AAD)/odontoid fracture from January to March 2020 and who received US-guided lumbar ESPB were retrospectively studied. All the necessary data were collected from the inpatient hospital, anesthesia, and the acute pain service records. A total of six patients received lumbar ESPB, of which one received a single shot injection, and the rest five had a catheter placement for postoperative analgesia. The average volume of intraoperative and postoperative bolus

Keywords

- erector spinae plane block

- occipitocervical fusion

- posterior-superior iliac spine

- postoperative analgesia was 27 (range: $15-30$ ) and 21 (range: $15-30$ ) $\mathrm{mL}$ of $0.2 \%$ ropivacaine, respectively. All patients achieved a unilateral sensory blockade ranging from L1 to L3 dermatomes. None of our patients had a numerical rating scale of $>4$ on movement at any time point during the first 48 hours except in one, in whom only a single shot bolus was given. No complications related to ESPB were noted. All were ambulated on the second postoperative day except one. The average length of hospital stay was 6 (range: 4-10) days. US-guided lumbar ESPB provides excellent analgesia for PSIS bone graft site pain and promotes early ambulation.
\end{abstract}

\section{Introduction}

Autologous bone graft from the posterior-superior iliac spine (PSIS) is still commonly used to augment bone healing and provide spine stability in patients undergoing occipitocervical fusion (OCF) and C1-C2 fusion for atlantoaxial dislocation

DOI https://doi.org/ $10.1055 / \mathrm{s}-0040-1721544$ ISSN 0976-3147.
(AAD)/odontoid fractures, especially in resource-poor settings. Pain at the graft site can range from moderate to severe, resulting in delayed postoperative ambulation and increased duration of hospital stay. ${ }^{1-3}$ Various modes of analgesia have been attempted to alleviate this graft site pain ranging from systemic morphine, paracetamol, and nonsteroidal anti-inflammatory 
drugs, local anesthetic infiltration as well as the various interfascial plane blocks. Recently, Gürkan and $\mathrm{Aksu}^{4}$ have reported the use of lumbar erector spinae plane block (ESPB) for graft site pain on a single case. Here, we report the analgesic efficacy of ultrasound (US)-guided lumbar ESPB for PSIS bone graft site pain in six patients undergoing OCF and $\mathrm{C} 1-\mathrm{C} 2$ fusion for odontoid fracture/AAD.

\section{Materials and Methods}

Patients who underwent OCF and C1-C2 fusion with PSIS bone grafting for AAD/odontoid fracture from January to May 2020 and who received lumbar ESPB for postoperative analgesia were retrospectively analyzed. Informed consent was obtained to perform the ESPB on the day before surgery. The demographic details and the preoperative diagnosis, presentations, and functional status were noted. All patients were anesthetized using a standard anesthesia protocol. After induction, the trachea was intubated with the aid of a fiberoptic bronchoscope (asleep technique). The patients were then positioned prone on a cerebellar headrest with skull pin traction. As per the existing neurosurgical protocols, cortical bone on the PSIS was harvested ( $3 \mathrm{~cm}$ [depth] $\times 5 \mathrm{~cm}$ [length]). Prior to incision and at closure, the neck wound was infiltrated with $10 \mathrm{~mL}$ of $0.2 \%$ ropivacaine. Fentanyl $(3-5 \mu \mathrm{g} / \mathrm{kg})$, morphine $(0.04-0.1 \mathrm{mg} / \mathrm{kg})$, and paracetamol $(20 \mathrm{mg} / \mathrm{kg})$ were given for intraoperative analgesia.

At the end of the surgical procedure, in the prone position (prior to turning the patient supine), ESPB was performed at L1-2 level. The high-frequency linear probe was placed in a sagittal plane at L1-2 level. An 18 G Touhy needle (in-plane approach) was introduced in a craniocaudal direction. Eight to ten $\mathrm{mL}$ of $0.2 \%$ ropivacaine was injected at each level (L1-2) below the erector spinae muscle. After creating a space in this facial plane, a $20 \mathrm{G}$ epidural catheter was placed between L1-2, and its correct placement was checked by injecting another $5-10 \mathrm{~mL}$ of $0.2 \%$ ropivacaine (total volume of $20-30 \mathrm{~mL}$ ) and the catheter was tunneled through the skin to prevent catheter displacement. The volume and concentration of the injected drug were decided after calculating the toxic dose based on their body weight. For those who had catheter placement for postoperative analgesia received a bolus of $20 \mathrm{~mL} 0.2 \%$ every 12th hourly for a period of 48 to 72 hours. As per our postoperative analgesia protocol, all patients received inj. paracetamol $1 \mathrm{~g}$ every 6th hourly and inj. diclofenac $75 \mathrm{mg}$ every 8 th hourly for postoperative analgesia (cervical site).

\section{Results}

A total of six patients received US-guided lumbar ESPB, of which one received a single shot injection (case no. 4), and the rest had a catheter placement for postoperative analgesia for 48-72 hours. The mean age of patients was 24 years (range: 11-49); with the male:female ratio of 5:1; the mean body mass index was 18.7 (range: 14-24). Three patients had a preoperative neurological deficit, and the rest had no deficit. The average volume of intraoperative and the postoperative bolus was 27 (range: 15-30) and 21(range: $15-30) \mathrm{mL}$ of $0.2 \%$ ropivacaine, respectively. All patients had a unilateral sensory blockade ranging from L1 to L3 dermatomal distribution, and none of them had motor blockade. The numerical rating scale (NRS) at the graft site (during rest and movement) at various periods for 48 hours (at arrival and 4,12, 24, and 48 hours) postoperatively is given in -Fig. 1. None of the patients had an NRS of $>4$ on movement at any time point except in case no. 4 in whom the catheter was not placed for postoperative analgesia. He had adequate pain relief for 12 hours following a single shot bolus, then he had moderate pain on movement with an NRS of 5 to 6 at the 24 and 48-hour time points. All patients were ambulated in and around the bed (with or without support) on the 2nd postoperative day except one, who had a cerebrospinal fluid (CSF) leak during the surgical procedure for which he was on bed rest for 5 days (case no. 6). The average length of the intensive care unit (ICU) and hospital stay was 1.1(range: 1-1.5) and 6 (range: $4-10$ ) days, respectively. None of them had complications related to the ESPB. One patient needed reoperation for correct placement of a displaced occipital screw (case no. 5), while other patient had a CSF leak requiring complete bed rest for 5 days that resulted in increase in the duration of hospital stay (case no. 6). The demographic details, preoperative diagnosis, intraoperative and postoperative bolus, ambulation time, the duration of ICU, and hospital stay are depicted in $\boldsymbol{-}$ Table $\mathbf{1}$.
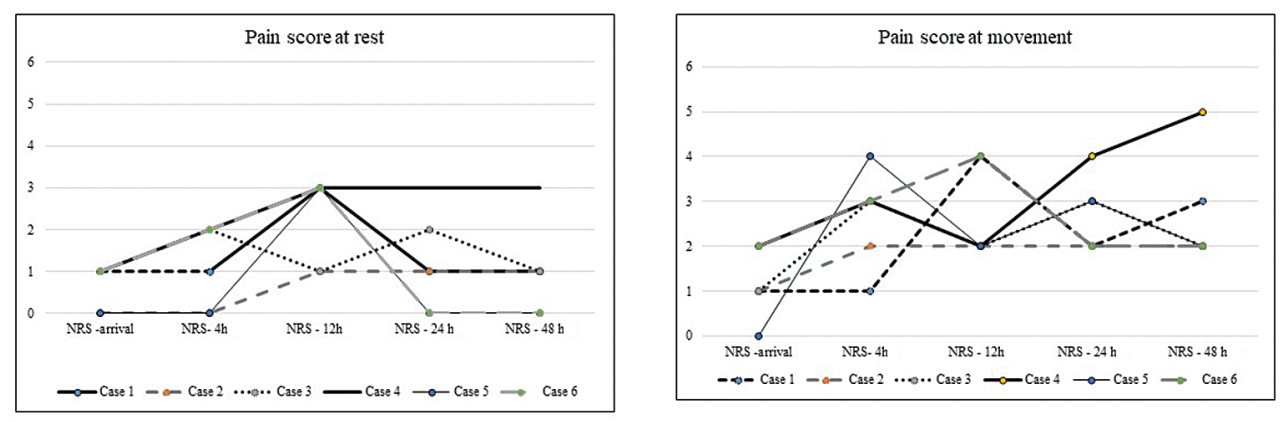

Fig. 1 Numerical rating scale (NRS) score at rest and movement during the first 48 hours postoperative period. 
Table 1 The demographic and intraoperative and the postoperative details of patients who received lumbar ESPB for PSIS graft site pain

\begin{tabular}{|c|c|c|c|c|c|c|c|c|c|}
\hline $\begin{array}{l}\text { Case } \\
\text { no }\end{array}$ & Age (y)/sex & BMI & $\begin{array}{l}\text { Preoperative } \\
\text { diagnosis }\end{array}$ & $\begin{array}{l}\text { Pre-op } \\
\text { Nurick } \\
\text { grading }\end{array}$ & $\begin{array}{l}\text { Volume and } \\
\text { concentration } \\
\text { of ropivacaine } \\
\text { given during } \\
\text { the intra-op } \\
\text { period }\end{array}$ & $\begin{array}{l}\text { Average } \\
\text { volume and } \\
\text { concentration } \\
\text { of ropivacaine } \\
\text { given during } \\
\text { the post-op } \\
\text { period }\end{array}$ & $\begin{array}{l}\text { Ambulation } \\
\text { time } \\
\text { (post-op } \\
\text { day) }\end{array}$ & $\begin{array}{l}\text { Length } \\
\text { of ICU } \\
\text { stay } \\
\text { (d) }\end{array}$ & $\begin{array}{l}\text { Length of } \\
\text { hospital stay (d) }\end{array}$ \\
\hline 1 & $16 / \mathrm{M}$ & 14 & $\begin{array}{l}\text { Traumatic } \\
\text { AAD with } \\
\text { os-odon- } \\
\text { toideum }\end{array}$ & 2 & $20 \mathrm{~mL}$ of $0.15 \%$ & $\begin{array}{l}15 \mathrm{~mL} \text { of } 0.15 \% \\
\text { Q12th hourly }\end{array}$ & 2 & 1 & 5 \\
\hline 2 & $49 / F$ & 24 & $\begin{array}{l}\text { Traumatic } \\
\text { AAD }\end{array}$ & 1 & $30 \mathrm{~mL}$ of $0.2 \%$ & $\begin{array}{l}20 \mathrm{~mL} \text { of } 0.2 \% \\
\text { Q12th hourly }\end{array}$ & 2 & 1 & 4 \\
\hline 3 & $29 / M$ & 20 & AAD & 1 & $30 \mathrm{~mL}$ of $0.2 \%$ & $\begin{array}{l}30 \mathrm{~mL} \text { of } 0.2 \% \\
\text { Q12th hourly }\end{array}$ & 2 & 1 & 4 \\
\hline 4 & $14 / M$ & 17 & $\begin{array}{l}\text { Marfan syn- } \\
\text { drome with } \\
\text { rotatory AAD }\end{array}$ & 0 & $30 \mathrm{~mL}$ of $0.2 \%$ & $N A^{a}$ & 2 & 1 & 4 \\
\hline 5 & $26 / M$ & 20 & $\begin{array}{l}\text { Traumatic } \\
\text { odontoid } \\
\text { fracture, C4 } \\
\text { facet, } \text { C5 body } \\
\text { fracture }\end{array}$ & 1 & $30 \mathrm{~mL}$ of $0.2 \%$ & $\begin{array}{l}20 \mathrm{~mL} \text { of } 0.2 \% \\
\text { Q12th hourly }\end{array}$ & 2 & 1.5 & $\begin{array}{l}10 \text { (reoperation } \\
\text { delayed the } \\
\text { discharge) }\end{array}$ \\
\hline 6 & $11 / \mathrm{M}$ & 17 & $\begin{array}{l}\text { Assimilation } \\
\text { of Atlas with } \\
\text { AAD }\end{array}$ & 3 & $20 \mathrm{~mL}$ of $0.2 \%$ & $\begin{array}{l}20 \mathrm{~mL} \text { of } 0.2 \\
\% \text { Q12th hourly }\end{array}$ & $\begin{array}{l}5 \text { (had CSF } \\
\text { leak-bed } \\
\text { rest for } 5 \mathrm{~d} \text { ) }\end{array}$ & 1 & $\begin{array}{l}9 \\
\text { (CSF leak delayed } \\
\text { the discharge) }\end{array}$ \\
\hline
\end{tabular}

Abbreviations: AAD, Atlantoaxial dislocation; CSF, cerebrospinal fluid; ESPB, erector spinae plane block; ICU, intensive care unit; PSIS, posterior-superior iliac spine.

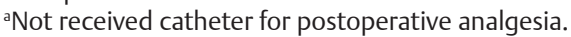

\section{Discussion}

Iliac crest autograft is often considered the gold standard for spine fusion procedures because of its vital properties of being osteoconductive, osteoinductive, and osteogenic. ${ }^{5}$ It is also a cost-effective alternative to expensive bone graft extenders, which have limited proof of efficacy. Nonetheless, pain at the graft site is undoubtedly added to the morbidity. Improper pain management at the graft site delays the postoperative recovery. Studies have shown that various interfascial blocks, such as the lumbar plexus block, are feasible options to provide analgesia for the graft site pain. ${ }^{6}$ However, they have many pitfalls such as being deep blocks, with steep learning curves and a higher risk of complications due to the close vicinity to vital structures. ESPB, being a superficial interfacial plane block, has been reported to provide adequate analgesia (for both, visceral and somatic pain) for various surgical procedures..$^{7-12}$ Though ESPB as a method of providing pain relief at the graft site has been described, the experience is sparse. ${ }^{4}$

In this case series, we would like to strengthen/establish the use of ESPB to provide analgesia for patients undergoing PSIS bone graft for spine fusion. With its easily recognizable sonoanatomy, vital structures along the needle path, and easy catheter insertion without hemodynamic changes, this block is simple and safe while providing better-quality analgesia.

In this era of enhanced recovery after surgery, the ESPB facilitates rapid recovery due to better pain management, early mobilization, and faster discharge from hospital. Based on the findings of this case series, lumbar ESPB has been made as a standard of care for providing analgesia at the PSIS graft site pain in our institution.

\section{Conclusion}

US-guided lumbar ESPB provides excellent analgesia for the PSIS bone graft site pain, thereby promoting early ambulation. Further prospective randomized control trials are needed to compare its analgesic efficacy with the other facial plane blocks.

\section{Author's Contributions}

All authors made material contributions to the handling of this case series and to the intellectual content of this article.

\section{Funding}

None.

\section{Conflict of Interest}

None declared. 


\section{References}

1 Armaghani SJ, Even JL, Zern EK, Braly BA, Kang JD, Devin CJ. The evaluation of donor site pain after harvest of tricortical anterior iliac crest bone graft for spinal surgery: a prospective study. Spine 2016;41(4):E191-E196

2 Blumenthal S, Dullenkopf A, Rentsch K, Borgeat A. Continuous infusion of ropivacaine for pain relief after iliac crest bone grafting for shoulder surgery. Anesthesiology 2005;102(2):392-397

$3 \mathrm{Kim} \mathrm{DH}$, Rhim R, Li L, et al. Prospective study of iliac crest bone graft harvest site pain and morbidity. Spine J 2009;9(11):886-892

4 Gürkan Y, Aksu C. Iliac crest bone graft donor site analgesia: a new indication for erector spinae plane block. Can J Anaesth 2019;66(3):338-339

5 Pirris SM, Nottmeier EW, Kimes S, O’Brien M, Rahmathulla G. A retrospective study of iliac crest bone grafting techniques with allograft reconstruction: do patients even know which iliac crest was harvested? Clinical article. J Neurosurg Spine 2014;21(4):595-600

6 Chin KJ, Chan V, Hebbard P, Tan JS, Harris M, Factor D. Ultrasound-guided transversalis fascia plane block provides analgesia for anterior iliac crest bone graft harvesting. Can J Anaesth 2012;59(1):122-123
7 Jain K, Jaiswal V, Puri A. Erector spinae plane block: relatively new block on horizon with a wide spectrum of application - a case series. Indian J Anaesth 2018;62(10):809-813

8 Melvin JP, Schrot RJ, Chu GM, Chin KJ. Low thoracic erector spinae plane block for perioperative analgesia in lumbosacral spine surgery: a case series. Can J Anaesth 2018;65(9):1057-1065

9 Singh S, Chowdhary NK. Erector spinae plane block an effective block for post-operative analgesia in modified radical mastectomy. Indian J Anaesth 2018;62(2):148-150

10 Adhikary SD, Pruett A, Forero M, Thiruvenkatarajan V. Erector spinae plane block as an alternative to epidural analgesia for post-operative analgesia following video-assisted thoracoscopic surgery: a case study and a literature review on the spread of local anaesthetic in the erector spinae plane. Indian J Anaesth 2018;62(1):75-78

11 Chin KJ, Adhikary S, Sarwani N, Forero M. The analgesic efficacy of pre-operative bilateral erector spinae plane (ESP) blocks in patients having ventral hernia repair. Anaesthesia 2017;72(4):452-460

12 Forero M, Adhikary SD, Lopez H, Tsui C, Chin KJ. The erector spinae plane block: a novel analgesic technique in thoracic neuropathic pain. Reg Anesth Pain Med 2016;41(5):621-627 